\title{
After-image fusion'
}

THOMAS BEHRENDT, M. D. AND ROGER RAYMOND

JEFFERSON MEDICAL COLLEGE, DEPARTMENT OF OPHTHALMOLOGY

A new aspect of the complex of after-image phenomena is described. It consists of the appearance of lines connecting initially separated after-images, if they are observed against a flickering background. These lines appear during the fading of the after-image. The after-images were produced by various configurations of Xenon flashes, the flickering background by a standard photostimulator. The phenomena was called after-image fusion not to be confused with binocular fusion. The report is qualitative. The main conclusion reached is the actual existence of the fusion phenomenon. Possible influences of conditioning or suggesting were debated and thought of small importance. The phenomenon casts some doubts on the simple mechanistic retinal origin of the after-images.

From antiquity Aristotle and later Goethe, Newton, and Descartes were intrigued by the fact that visual activity continues after retinal stimulation has ceased. Purkinje is considered to have been the first to study after-images comprehensively using the tools of modern physiologic investigation. In spite of the amount of information gathered about after-images and allied phenomena, little is know about their mechanism, and even the site of their formation is disputed. Since Craik, the consensus is that they are of retinal origin. Howard, however, demonstrated a phenomenon indicating a central component.

While working routinely with after-images, a new phenomenon has been discovered which could not be found described in the general literature. Essentially, this phenomenon consists of the perception of lines connecting separate but simultaneously produced afterimages observed against a flickering background. For simplicity, this phenomenon has been called fusion of the after-image; not to be confused with binocular fusion. The present report covers a qualitative study of this new aspect of the after-image.

Method

The experiments were conducted in a room with walls evenly painted a light green. A Grass photostimulator (Model PS2) was the only source of illumination in the room while observing the fusion. This photostimulator set at a frequency of 11 flashes per sec. and at its output intensity of 4 (arbitrary scale on Model PS2) provided a flickering field.

Two types of flash-stimulators were used to produce the after-images. The first consisted of a conventional linear electronic flash tube $32 \mathrm{~cm}$ long with a $7 \mathrm{~mm}$ quartz body. It was powered by a $23.4 \mathrm{mfd}$ capacitor charged to 2000 volts. Flashes of variable lengths, separated by variable gap sizes, were obtained by adequately masking this tube with black tape (Fig. 1). The second flash stimulator consisted of four con-

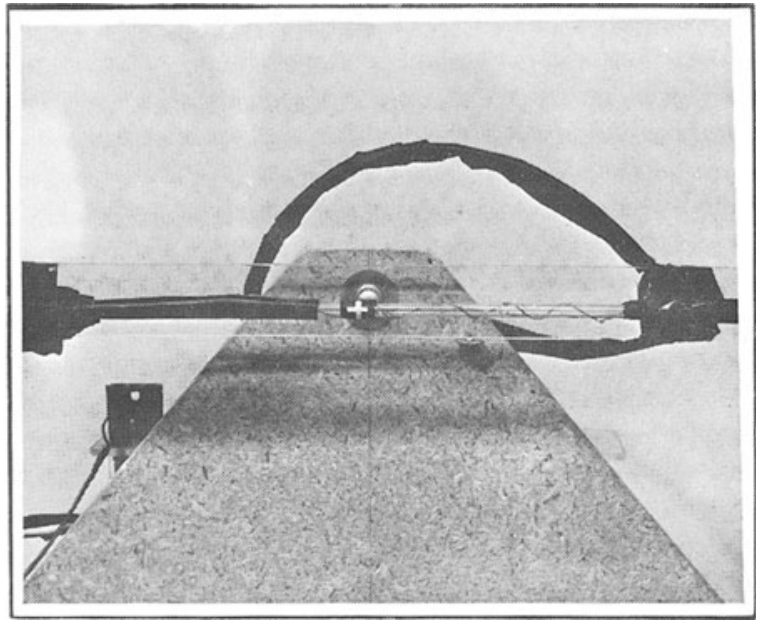

Fig. 1. Linear electronic flash stimulator covered with black tape to produce variable stimuli.

ventional helicoidal flash tubes attached to the back of a board. Their light output was limited by $2.5 \mathrm{~cm} \emptyset$ holes arranged at the corners of a $15 \mathrm{~cm}$ square. Each flash was powered by $1100 \mathrm{mfd}$ charged to 450 volts wired to ignite simultaneously. Light was diffused by inserting a paper screen between the board and the flash tubes. Various patterns of flash stimuli were obtained by covering the holes with black tape.

A procedure was standardized as follows: For the first part of the experiment, the linear electronic flash stimulator was placed $60 \mathrm{~cm}$ from a wall, while the $\mathrm{S}$, sitting $150 \mathrm{~cm}$ from this wall, was instructed to fixate monocularly (with the other eye covered) at the middle of the black tape in the center of the flash stimulator. The photostimulator, placed $50 \mathrm{~cm}$ behind the $S$, was aimed at the wall in front of the S. Immediately after the flash stimulator was ignited, the $S$ was asked to watch his after-images in the flickering field on the wall. He was also told to look for lines possibly appearing between the after-images. At the outset, the phenomenon was demonstrated to him under an extremely favorable condition (two long $15 \mathrm{~cm}$ flashes separated by a rather small, $1 \mathrm{~cm}$ gap) in which fusion was known to occur.

He was subjected to a series of stimulations with two $2 \mathrm{~cm}$ flashes. These flashes were successively separated by distances of $1,2,4,8,16$, and $32 \mathrm{~cm}$. This procedure also was performed with a reversed sequence of gap sizes. A similar series was presented with $1 \mathrm{~cm}$ flashes.

In the second part of the experiment, the helicoidal flash stimulator was substituted for the linear flash stimulator. Other conditions were unchanged. The $\mathrm{S}$ 
was instructed to fixate monocularly at fixed points on the board while different patterns of stimuli were presented.

An interval of approximately 5 min. was maintained between observations. Room lights were turned on during this time.

\section{Results}

The actual description of the fusion phenomenon by the observers varied slightly. Some described it as a white, others as a blue, luminous line. The primary repeatable observation was that when the actual after-images started to fade, a single continuous straight line bridged the gap between previously separated after-images. It is important to note that the after-images themselves did not move and that fusion was the occurrence of a line bridging the gap between them (Figs. 2 and 3 ).

The first phase of the experiment with the linear flash-stimulator demonstrated that there was a range of flash sizes and gaps in which fusion occurred and a range in which fusion did not occur. Generally speaking, the limit for fusion was reached by decreasing the size of the flash and increasing the size of the gap. The point where the $\mathrm{S}$ stopped seeing fusion when the size of the gap was increased after starting from definite perception often coincided with the point where the $S$ began seeing fusion when the gap was decreased after starting from no perception of fusion.

With the second helicoidal flash-stimulator, results varied. With the two holes uncovered, most Ss saw fusion between the two after-images. With three, a triangular configuration was observed. With four, however, the responses varied from $S$ to $S$ and with the direction of gaze. By fixating at the center of the

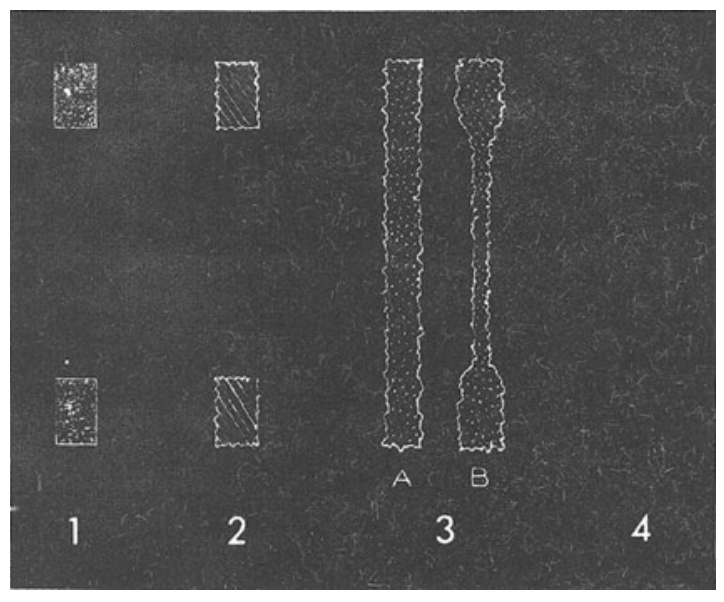

Fig. 2. After-image and fusion sequence produced by linear stimulator seen on a flickering background. (1) After-images immediately after flash. (2) After-images starting to fade. (3) After-image fusion: (A) Width equal to original after-image (B) Narrower than original after-image. (4) Disappearance.

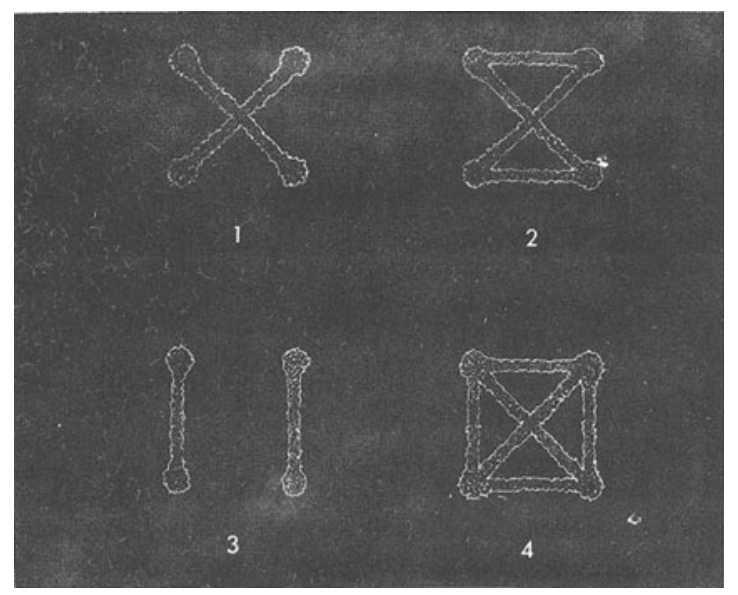

Fig. 3. Different patterns of fusion produced by four spots seen on a flickering background with different points of fixation.

square many Ss reported seeing fusion diagonally connecting the after-images, thus forming an $\mathrm{X}$ configuration. While fixating on the side of the square many parallel lines were reported frequently. At least one fusion line consistentiy crossed the fixation point.

\section{Discussion}

As in all subjective phenomena the concept of learning, conditioning, and suggestion had to be considered. Learning did play some role because it was initially necessary to demonstrate fusion under very favorable circumstances.

However, conditioning and suggestion were ruled out as major factors because the Ss had substantially identical transition points between fusion and no fusion, whether approached by increasing or decreasing the gap-size.

Some Ss were confused between lines normally seen crossing a flickering field and the fusion image. This was avoided by reminding them that these lines cross the whole field, while fusion extends only between two after-images.

While these experiments were conducted to explore the after-image fusion phenomenon, their results cast some doubt on a purely retinal mechanism of afterimages.

\section{References}

Adler, F. H. Physiology of the eye. St. Louis: C. V. Mosby, 1959. P. 645 .

Craik, W. Origin of visual after-images. Nature, London, 1940 , $145,512$.

Davson, H. The eye. New York: Academic Press 213, 1962.

Duke-Elder, S. Textbook of ophthalmology. London: Henry Kimpton, 1933.

Howard, I. P. Some new subjective phenomenon apparently due to intraocular transfer. Nature, 1959, 184, 1516.

Note

1. This investigation was supported by NINDB Grant NB 5456-01. 